\title{
Philosophiques
}

\section{Zeimbekis, John. Qu'est-ce qu'un jugement esthétique? Vrin, coll. Chemins philosophiques, 2006, 128 p.}

\section{Mélissa Thériault}

Volume 34, numéro 2, automne 2007

URI : https://id.erudit.org/iderudit/017430ar

DOI : https://doi.org/10.7202/017430ar

Aller au sommaire du numéro

Éditeur(s)

Société de philosophie du Québec

ISSN

0316-2923 (imprimé)

1492-1391 (numérique)

Découvrir la revue

Citer ce compte rendu

Thériault, M. (2007). Compte rendu de [Zeimbekis, John. Qu'est-ce qu'un jugement esthétique? Vrin, coll. Chemins philosophiques, 2006, 128 p.]

Philosophiques, 34(2), 421-425. https://doi.org/10.7202/017430ar d'utilisation que vous pouvez consulter en ligne.

https://apropos.erudit.org/fr/usagers/politique-dutilisation/ 
mécanique quantique, mais il est certain qu'il n'y a pas de champ magnétique sans dipôle magnétique, et pas de dipôle magnétique sans un support quelconque (aimant, courant électrique, corps céleste, etc.), de même qu'il n'y a pas de champ magnétique actuel sans électrons qui se meuvent d'un pôle à l'autre. En somme, on n'observe point de champ magnétique sans support.

Plus loin dans le même chapitre, Rescher présente deux nouveaux exemples: la chute de la demande économique et l'érosion de la ligne côtière. Or, de la même façon, la chute de la demande économique est une statistique induite à partir d'un nombre déterminé d'individus, et l'érosion de la ligne côtière est toujours constituée d'un nombre déterminé de processus spécifiques de dissociation, ayant chacun pour support un nombre déterminé de grains de sable (ou autre), spatiotemporellement localisables.

Ainsi, parce que les thèses générales de la philosophie du processus reposent sur la thèse de la séparabilité de certains processus et que Rescher n'a pas démontré que ceuxci existent effectivement de façon séparée, alors la philosophie (rescherienne) du processus ne repose sur aucun fondement satisfaisant, du moins dans sa version ontologique, dite "forte» (et dans la mesure où la version épistémologique, dite "faible ", reposerait sur les mêmes fondements, alors elle serait tout aussi problématique). En effet, sans la thèse de l'existence de processus impropres, celles de la primauté causale et du caractère fondamental des processus se dissolvent, car pour être premier ou fondamental par rapport à autre chose il faut d'abord que l'un puisse se trouver sans l'autre.

La traduction est généralement conforme. Toutefois la reddition de owned et unowned par «propre» et «impropre» est malheureusement cryptique. De plus, le choix surprenant de "procès " plutôt que "processus » n'est nulle part sérieusement motivé. Weber se contente d'une note dans le lexique où il écrit que process est "parfois traduit, pour des raisons euphoniques, [par] "processus" » (p. 276). Or "processus » est préférable pour des raisons plus sérieuses que la simple euphonie. Parmi ces raisons, notons d'abord que le premier sens de "procès" est juridique, de sorte que l'expression « ontologie du procès » pourrait être méprise pour une espèce de l'ontologie du droit et, ensuite, que l'usage déjà établi du terme processus dans le vocabulaire francophone de la philosophie n'a rien de problématique et qu'il est donc superficiel de lui chercher un substitut.

FRÉDÉRIC TREMBLAY

SUNY at Buffalo et IFOMIS (Saarbrücken)

\section{Zeimbekis, John. Qu'est-ce qu'un jugement esthétique? Vrin, coll. Chemins philosophiques, 2006, $128 \mathrm{p}$.}

Cette nouvelle parution relève le défi de présenter une notion incontournable qui a alimenté les querelles et débats en esthétique au dix-huitième siècle. En effet, la notion de jugement esthétique, conçue à l'époque en tant qu'affirmation portant sur la beauté d'un objet ( «x est beau », "y est élégant », etc.) a alimenté des querelles épistémologiques sur la nature et le statut de tels jugements. Par exemple : à quelles conditions puis-je dire, si j'affirme que " $\mathrm{x}$ est beau », que mon affirmation est vraie? On l'aura deviné : l'enjeu est de déterminer à quelles conditions ce type d'affirmation peut avoir un caractère objectif et par conséquent à quelles conditions on pourrait en faire la pierre d'assise d'un discours sur le beau et sur la notion de plaisir. Le traitement 
philosophique de cette notion a connu des suites multiples. Si Kant a proposé une analyse suffisamment convaincante aux yeux de certains pour qu'ils élèvent sa proposition au statut de dogme (sous la forme du jugement de gồt), d'autres, en revanche, ont relégué le jugement esthétique au rayon de l'indiscutable en raison de son caractère (en apparence du moins) hautement subjectif. Si « des goûts et des couleurs, il ne faut pas discuter », comment prétendre construire un discours argumentatif fondé sur des jugements portant, justement, sur l'appréciation de ceux-ci?

L'ouvrage est divisé en deux sections : dans la première, la problématique est présentée et analysée d'un point de vue général. La seconde est une analyse de textes portant sur un extrait de "De la règle du goût " de Hume (la section où il est question de la figure de l'homme de goût est toutefois amputée) et un texte plus récent de Malcolm Budd traduit sous le titre "Jugements esthétiques, principes esthétiques et propriétés esthétiques ». La toute première phrase de l'ouvrage est une mise en garde non superflue : la possibilité même de l'existence du jugement esthétique est remise en question. À cette fin, l'auteur explique comment l'expression s'est imposée dans le langage et précise qu'elle est peut-être, justement, en quelque sorte un abus de langage puisque "il est possible que les jugements esthétiques ne soient pas des jugements en un sens substantiel et philosophiquement admis du terme " et que l'on "ne peut exclure qu'ils soient fondés sur une forme d'appréciation qui relève de la raison pratique» (p. 7). Autrement dit, bien que cette manière d'exprimer la problématique propre au langage des Lumières soit entrée dans les conventions langagières, la fréquence de son emploi ne met pas la notion à l'abri de la remise en question, et le débat qui l'entoure est loin d'être clos. Toutefois, c'est l'ambition de plusieurs philosophes de montrer «qu'il existe des jugements proprement esthétiques » et dans quels cas ils peuvent être bien fondés, notamment en tentant de cerner les conditions de possibilité des jugements esthétiques ainsi que les circonstances dans lesquelles celles-ci pourraient être satisfaites, ne seraitce qu'en principe (p. 8).

L'ouvrage donne la parole à deux figures historiques incontournables de la discipline (Hume et Kant) ainsi qu'à un interlocuteur plus récent (Frank Sibley) en établissant les parallèles et distinctions qui s'imposent entre leurs solutions respectives. On souligne aussi à juste titre que la problématique des jugements esthétiques est revue dans le contexte actuel à la lumière de spécialisations telles que la psychologie cognitive, l'épistémologie et la philosophie de la perception plutôt que dans le cadre d'une philosophie de l'art, qui ne recoupe aujourd'hui que partiellement les préoccupations qui étaient celles de l'esthétique philosophique classique. Comme le rappelle à juste titre Zeimbekis, tous les jugements portant sur les œuvres ne sont pas pour autant des jugements esthétiques (p. 13). On s'attarde aussi à tracer les grandes lignes du contexte historique dans lequel s'insérait la notion, et ce, d'une façon claire et détaillée (p. 8-9). Par exemple, si la notion de beauté peut nous apparaître comme un concept central dans l'esthétique du dix-huitième siècle, elle était loin d'être dominante ( les notions de sublime ou de grâce occupaient à certains moments des rôles au moins aussi importants (p. 9). Le concept de beauté n'est plus incontournable non plus en esthétique contemporaine et encore moins en philosophie de l'art, surtout depuis que le rapport entre art et beauté s'est effrité avec l'avènement de la modernité artistique. Zeimbekis souligne la variété des concepts esthétiques employés ainsi que les écueils potentiels qui se présentent lorsqu'on se limite aux concepts génériques (beau/laid) possédant une lourde charge évaluative (p. 10). Il précise que l'usage de l'expression «expérience esthétique » n'implique pas la croyance en une sorte d'expérience particulière qui serait ainsi désignée: 
une expérience esthétique est une expérience qui nous porte à dire d'une chose que celle-ci est élégante (p. 11).

On effectue aussi un nécessaire retour sur la distinction entre propriétés esthétiques et propriétés artistiques : souvent, les affirmations à propos des œuvres d'art portent sur des qualités relevant du contenu représentationnel ou du potentiel symbolique (p. 13) plutôt que des propriétés esthétiques. En ce sens, ce qu'on dit des œuvres d'art prend rarement la forme d'un jugement esthétique, même si de telles affirmations sont souvent traitées par l'esthétique en tant que discipline (p. 14). Cependant, puisque l'ouvrage doit se limiter aux jugements esthétiques, l'auteur s'attarde peu à cette nuance pourtant cruciale, pour effectuer plutôt un rappel sur les premières théories autour du jugement esthétique (mises de l'avant notamment par Hutcheson). Celles-ci postulaient l'existence d'un sens interne, le "sens esthétique ", c'est-à-dire une "capacité passive d'avoir des impressions, pour expliquer comment nous jugeons esthétiquement les objets» (p. 15). Celle-ci agirait comme intermédiaire entre les sensations extérieures reçues par les sens et le sentiment de plaisir/déplaisir éprouvé par le sujet (p. 15) et permettrait en quelque sorte de protéger les jugements esthétiques de la contingence (p. 16). Pourtant, les jugements esthétiques en tant que "symptômes observables de nos réactions esthétiques " ne font pas consensus, ce qui met à mal cette hypothèse chère aux empiristes (p. 17). Viennent ensuite les théories physiologiques, dont celle de Burke (qui, au dix-huitième siècle, relie les expériences esthétiques à des instincts) ainsi que sa variante contemporaine neurologique, mise de l'avant par V. Ramachandran, qui s'appuie sur une réflexion autour des mécanismes de la cognition perceptive et cherche à baser l'objectivité des jugements sur une sorte de réalisme biologique (p. 21). Burke formule sa théorie en termes de sentiments esthétiques (sentiment de sublime, de beau) et ses limites sont, selon Zeimbekis, surmontées par la variante contemporaine de Ramachandran qui prend assise sur des notions évolutionnistes et tente d'expliquer les réactions esthétiques en "analysant le fonctionnement de notre physiologie perceptive », ce qui permettrait de générer des « lois de l'art » que les artistes utiliseraient consciemment ou non pour stimuler les régions visuelles du cerveau (p. 27). Malheureusement, il n'est pas mentionné si la thèse de Ramachandran vise à s'appliquer à autre chose qu'aux arts visuels : on présente cependant les différentes objections qui ont été faites à cette approche, dont sa circularité (p. 29).

L'essentiel de l'analyse de Zeimbekis porte sur la formulation de la problématique par Hume qui a abordé la question sous un angle nouveau : en cherchant à soumettre le concept de jugement esthétique à l'épreuve du scepticisme, il tente de le reformuler sur des assises résistant à ses propres objections (p. 33) afin de répondre à la question: "comment, et sous quelles conditions, un jugement fondé sur un sentiment peut-il être valide?" (p. 37). Certaines distinctions s'imposent alors, par exemple entre jugements objectifs et jugements portant sur des propriétés objectives (p. 38). Après avoir déterminé à quelles conditions un jugement pouvait être objectif, il importe ensuite de voir "s'il y a bien des faits indépendants qui puissent conférer aux jugements esthétiques le statut de représentations vraies ou fausses" (p. 39). Une partie de la solution proposée par Hume consiste à attribuer aux désaccords liés au goût aux capacités de discernement du sujet: le critique idéal est plus sensible aux nuances et détails parce qu'il a affiné son goût, notamment par la pratique (p. 39). Mais malgré ses tentatives de situer objectivement le jugement de goût, Hume est relégué au camp conventionnaliste dès lors qu'il se résigne à croire que les faits esthétiques ne peuvent être connus que par consensus (p. 43), et on pourrait être tenté, selon Zeimbekis, de lui reprocher de n'avoir comme solution 


\section{Philosophiques / Automne 2007}

de rechange au problème du subjectivisme individuel qu'une proposition de substitution par un subjectivisme de groupe (p. 44). C'est alors que sont présentées les solutions de rechange proposées par Kant aux problèmes auxquels Hume n'aurait pas proposé de solution satisfaisante.

Si Hume a tenté de donner au jugement esthétique le statut d'un jugement de faits pour surmonter le défi subjectiviste (position selon laquelle un jugement de sentiments n'est ni vrai ni faux) (p. 44), Kant a procédé selon le cheminement inverse en acceptant le caractère subjectif du jugement esthétique mais en lui attribuant une forme de validité particulière (p. 45). C'est le concept même de jugement qui est ainsi transformé, ce qui aura des répercussions au-delà de l'esthétique kantienne déployée dans Critique de la faculté de juger. La validité du jugement esthétique dépend de certaines conditions cognitives très précises (p. 45), conditions qui sont explicitées par Kant et distinguées des circonstances où l'on pourrait être porté à croire à tort que notre jugement de goût est bien fondé. La faculté de juger n'est toutefois pas confinée au jugement esthétique et "est également requise pour accomplir d'autres tâches cognitives " (p. 45). Quant à l'origine de la satisfaction esthétique, elle est résumée ainsi: "mon activité cognitive est satisfaisante quand je contemple la forme parce que la forme, étant finalisée, convient aux principes de la faculté de juger, qui gouverne mes tentatives perceptives de cerner des formes finalisées » (p. 47) : c'est en présence de ce type de satisfaction que l'on qualifie l'objet l'ayant provoquée de "beau ». Kant cherche à expliquer qu'il y a des jugements auxquels on peut attribuer une forme de validité sans en faire pour autant des jugements de faits (p. 47). Le jugement de goût qui, dans le contexte humien était un jugement de sentiment et non factuel, possède selon lui ces caractéristiques : il est non conceptuel, ne peut attribuer de propriété à l'objet et est fondé sur un sentiment de plaisir (p. 49), mais il est valable sur le plan intersubjectif (p. 50). La théorie proposée par Kant comporte aussi ses points de tension, notamment en ce qu'elle oblige à accepter un postulat quant à l'existence d'idées esthétiques, principes régulateurs de réactions esthétiques inhérents à la faculté de juger (p. 50). L'auteur relève alors que la prémisse des idées esthétiques "joue exactement le même rôle que le postulat métaphysique de Hume» (p. 51) et renvoie les deux théories à dos en leur reprochant un point commun, à savoir, de fonder la normativité des jugements esthétiques "sur des postulats douteux", en l'occurrence, selon Zeimbekis, des faits invérifiables dans le cas de Hume et des principes inconnaissables du côté de Kant (p. 56).

L'auteur effectue ensuite une parenthèse visant à faire un état des lieux quant aux différents modèles du jugement esthétique : l'approche la plus courante est de poser le problème en tant que "relation entre un particulier et un concept évaluatif ", ce qui inclut bien sûr les approches issues de l'empirisme, du réalisme et de la philosophie kantienne pour lesquelles l'auteur avoue son parti pris (p. 57). Il signale aussi l'existence d'un autre type d'approche, représenté par les théories qui "ne portent pas sur le jugement esthétique mais sur le contenu des cuvres d'art ", parmi lesquelles celles de Hegel et d'Adorno font figure d'illustres exemples (p. 57). À cela s'ajoutent les théories relativistes qui «critiquent le concept même de jugement esthétique » : par exemple, Genette fait usage du concept d'expérience esthétique mais ne reconnaît pas la validité du jugement qui pourrait être formulé à la suite de cette expérience par le sujet (p. 57). Un autre modèle regroupe les théories "qui défendent la possibilité qu'il existe des jugements esthétiques bona fide» (p. 58) et qui se heurtent aux objections classiques évoquées tout au long de l'ouvrage. Parmi celles-ci, la variante proposée par F. Sibley dans les années soixante, qui relève trois types de concepts circonscrits par des frontières 
souvent floues : concepts non esthétiques, esthétiques et évaluatifs (p. 59). La théorie de Sibley repose sur une analyse du rapport qu'ils entretiennent les uns aux autres et comporte le mérite de mieux répondre aux critiques de relativisme qu'on pouvait faire aux théories évoquées précédemment (p. 68). Mais elle n'est pas exempte non plus de difficultés, soulignons par exemple le caractère culturellement relatif des concepts esthétiques (p. 71).

La seconde partie de l'ouvrage, composée du commentaire des textes de Hume et de Budd, permet de situer les notions vues précédemment dans une perspective plus générale. Puisque la théorie humienne avait été présentée plus en détail auparavant, Zeimbekis propose un rappel de l'opposition entre conception relativiste (motivée par un constat empirique) et réaliste telle que présentée par Hume (p. 80-81). On insiste aussi sur les préoccupations «méta-esthétiques » de Hume, moins intéressé par la définition des concepts esthétiques que par une discussion autour de leur légitimité (p. 84) ainsi que sur les limites de la forme de réalisme proposée par Hume (p. 90). Le texte de Budd consiste pour sa part en un court extrait qui discute une interprétation contemporaine de Hume, coupé toutefois du contexte de discussion dans lequel il s'insérait. On cherche à y repousser le relativisme des jugements esthétiques en défendant l'existence de "principes esthétiques logiquement faibles» (p. 108). Le principal mérite de l'extrait choisi est de servir de prétexte à l'introduction de certaines figures contemporaines dont Alan Goldman et Eddy Zemach.

En conclusion, disons que cette publication relève le défi de rendre compte d'une problématique qui a une longue histoire tout en réussissant à résister à la tentation du parti pris et en sachant mettre en valeur la problématique. La commande implique en soi une grande neutralité de l'auteur, exigence qui a été respectée admirablement, d'autant plus que le format imposé par la collection oblige un traitement très schématique qui ajoute à la difficulté de la tâche. L'auteur a également su mettre en perspective les théories autour du jugement esthétique : bien que populaires chez les historiens qui s'intéressent à l'esthétique, ou dans leurs variantes contemporaines, elles ne sont pas les seules approches possibles, et la pertinence même de traiter de la notion de jugement esthétique a été remise en question par plusieurs. Notons que ce petit volume remplira sa fonction principalement auprès d'un lectorat déjà familier et sympathique aux théories issues de l'empirisme. Mais ce défaut a une contrepartie positive: on va à l'essentiel, ce qui permet de comprendre les enjeux de ce problème historique qui suscite toujours l'intérêt de plusieurs.

MÉLISSA THÉRIAULT

UQAM/Université de Provence

\section{Jean-François Filion, Dialectique et matière. La conceptualité incon- sciente des processus inorganiques dans la Philosophie de la nature (1830) de Hegel, Québec, Les Presses de l'Université Laval, 2007, 346 p.}

Cet ouvrage impose d'abord par son ambition. Reconnaissons d'emblée que la philosophie de la nature n'est ni la partie la plus connue du système hégélien ni encore moins la plus considérée. Or, dans cette étude, qui constitue une version remaniée de sa thèse de doctorat, Jean-François Filion se propose non pas simplement de réhabiliter la philosophie de la nature de Hegel — d'autres auteurs s’y sont déjà 